\title{
Desenvolvimento sustentável impulsionado pelas tecnologias na agricultura
}

\author{
Sustainable development driven by technologies in agriculture \\ El desarrollo sostenible impulsado por las tecnologías en la agricultura
}

Recebido: 04/08/2021 | Revisado: 13/08/2021 | Aceito: 14/08/2021 | Publicado: 16/08/2021

Taiane Aparecida Ribeiro Nepomoceno
ORCID: https://orcid.org/0000-0003-3291-4221
Universidade Estadual do Oeste do Paraná, Brasil
E-mail: taiane.nepomoceno@ @nioeste.br
Eduarda Remor Bastos
ORCID: https://orcid.org/0000-0001-7114-5838
Universidade Tecnológica Federal do Paraná, Brasil
E-mail: eduardaremorbastos@yahoo.com.br

\begin{abstract}
Resumo
O principal foco desta pesquisa é a tecnologia digital na agricultura, também definida como agricultura 4.0, e sua relação com os Objetivos do Desenvolvimento Sustentável contemplados na Agenda 2030, da ONU. Como método, foi realizada uma revisão sistemática de literatura, a fim de buscar elementos que evidenciam de que forma o tema vem sendo descrito em artigos científicos publicados nos últimos dez anos. Após a varredura, foram selecionados 15 estudos publicados nos anos de 2018, 2019, 2020 e 2021. Esses indicativos evidenciam o crescimento de publicações sobre agricultura 4.0. Percebeu-se entre os artigos, ênfase aos estudos relacionados com o desenvolvimento de modelos de tecnologia digital para culturas agrícolas específicas, assim como estudos que tencionavam compreender as relações entre o uso e as dificuldades de implementação dessas, no contexto produtivo. De forma unânime, todos os estudos selecionados se relacionam com o $9^{\circ}$ objetivo da Agenda da ONU, que têm como principal meta, a construção de processos produtivos inclusivos, sustentáveis, resilientes, com enfoque na inovação. Neste caso, a inovação tem grande relação com o digital na agricultura. Os resultados, apontam ainda para evidências da necessidade de formulação de políticas públicas eficientes, de incentivo e continuidade, para grandes e pequenos produtores; sugerem que este é um dos principais aspectos a serem considerados, tendo em vista o estabelecimento agrícola, sua cultura e modo de produção.
\end{abstract}

Palavras-chave: Agricultura 4.0; Sustentabilidade; Acesso; Inovação.

\begin{abstract}
The main focus of this research is digital technology in agriculture, also defined as agriculture 4.0, and its relationship with the Sustainable Development Goals included in the UN's 2030 Agenda. As a method, a systematic literature review was carried out, in order to seek elements that show how the topic has been described in scientific articles published in the last ten years. After the scan, 15 studies published in 2018, 2019, 2020 and 2021 were selected. These indicators show the growth of publications on agriculture 4.0. Among the articles, emphasis was placed on studies related to the development of digital technology models for specific agricultural crops, as well as studies that intended to understand the relationship between their use and the difficulties of implementing these in the productive context. All selected studies are unanimously related to the 9th objective of the UN Agenda, whose main goal is the construction of inclusive, sustainable, resilient productive processes, with a focus on innovation. In this case, innovation has a strong relationship with the digital in agriculture. The results also point to evidence of the need to formulate efficient public policies, of incentives and continuity, for large and small producers; suggest that this is one of the main aspects to be considered, considering the agricultural establishment, its culture and mode of production.
\end{abstract}

Keywords: Agriculture 4.0; Sustainability; Access; Innovation.

\section{Resumen}

El foco principal de esta investigación es la tecnología digital en la agricultura, también definida como agricultura 4.0, y su relación con los Objetivos de Desarrollo Sostenible incluidos en la Agenda 2030 de la ONU. Como método, se realizó una revisión sistemática de la literatura, con el fin de buscar elementos que muestren cómo se ha descrito el tema en los artículos científicos publicados en los últimos diez años. Después de la exploración, se seleccionaron 15 estudios publicados en 2018, 2019, 2020 y 2021. Estos indicadores muestran el crecimiento de las publicaciones sobre 
agricultura 4.0. Entre los artículos, se hizo énfasis en estudios relacionados con el desarrollo de modelos de tecnología digital para cultivos agrícolas específicos, así como estudios que pretendieron comprender la relación entre su uso y las dificultades de implementarlos en el contexto productivo. Todos los estudios seleccionados se relacionan unánimemente con el noveno objetivo de la Agenda de la ONU, cuyo principal objetivo es la construcción de procesos productivos inclusivos, sostenibles, resilientes, con foco en la innovación. En este caso, la innovación tiene una fuerte relación con lo digital en la agricultura, los resultados también evidencian la necesidad de formular políticas públicas eficientes, de incentivos y continuidad, para grandes y pequeños productores; sugieren que este es uno de los principales aspectos a considerar, considerando el establecimiento agrícola, su cultura y modo de producción.

Palabras clave: Agricultura 4.0; Sostenibilidad; Acceso; Innovación.

\section{Introdução}

Todos os anos, novas políticas, conceitos e tecnologias passam a ser incorporadas a diferentes contextos e processos produtivos. Neste sentido, a agricultura também recebe novas reorientações que podem otimizar a produção e melhorar a qualidade de vida no campo. O mundo globalizado, por sua vez, ao mesmo tempo, em que demonstra evoluir na criação de tecnologias e outros recursos, aponta para a necessidade de repensar um modelo de desenvolvimento mais justo e equitativo, onde a resiliência do ambiente seja respeitada e a sustentabilidade o seu elemento primordial.

Com base nisso, o uso de tecnologias digitais na agricultura representa uma inovação do ponto de vista estratégico, para o aprimoramento dos processos produtivos e de atendimento às demandas atuais. Entretanto, acredita-se que não é possível que a sociedade como um todo avance, se ainda houver fome, pobreza, saneamento inadequado, produções que gerem degradação socioambiental, dentre outros. Por este motivo, o principal foco desta pesquisa é a tecnologia digital na agricultura e sua relação com os Objetivos do Desenvolvimento Sustentável ${ }^{1}$.

Destarte, a agricultura digital, também definida como agricultura 4.0, vai muito além da inserção de novas tecnologias nos processos produtivos, ela poderá contribuir com a atenuação do uso de agrotóxicos, fertilizantes e consumo de água. As tecnologias digitais poderão viabilizar, de forma mais segura, as aplicações em áreas específicas, com dosagens mínimas; além de auxiliar na minimização do uso dos recursos naturais, melhoria da qualidade de vida dos produtores, aumento da produtividade, maior compartilhamento de informações, melhoria das cadeias produtivas e, possibilitando uma maior integração entre o meio rural e urbano (Clercq; Vats \& Biel, 2018).

De forma geral, a agricultura digital, emprega recursos de ponta, rede de sensores, computadores de alto desempenho, automação, bigdata, internet das coisas, computação em nuvem e muitos outros métodos, capazes de auxiliar no processamento de dados e na elaboração de sistemas eficientes de suporte, para a tomada de decisões de manejo. Logo, ela poderá certamente elevar os níveis de eficiência de insumos, reduzindo altos custos de mão de obra e, principalmente, minimizando os impactos causados ao meio ambiente (Massruhá \& Leite, 2017).

Embora já se evidencie a relação da agricultura digital para a melhoria de diversas tendências pertinentes ao Desenvolvimento Sustentável, ainda são poucos os estudos com enfoque à agricultura 4.0 e sua relação com os Objetivos do Desenvolvimento Sustentável da ONU. Assim, é fundamental, em vista disso, o desenvolvimento de uma varredura bibliográfica entre essas temáticas e seus elementos fundantes, visto que juntas constituem um sistema emergente e de grande importância para trilhar o caminho rumo a construção de uma sociedade mais sustentável.

Desta forma, este artigo tem o objetivo de evidenciar de que forma o tema, agricultura digital vem sendo descrita em artigos científicos publicados ao redor do mundo e sua relação com os Objetivos do Desenvolvimento Sustentável, da ONU.

\footnotetext{
${ }^{1}$ Os 17 Objetivos do Desenvolvimento Sustentável estão contemplados na Agenda 2030, da ONU. Para mais informações, consultar o link $<$ http://www.agenda2030.com.br/>
} 


\section{Metodologia}

Este estudo corresponde a uma revisão sistemática de artigos publicados, relacionados à agricultura digital e ao alcance do desenvolvimento sustentável. Por isto, a presente pesquisa pode ser classificada como exploratória, de natureza quali-quantitativa, realizada a partir das instruções da ferramenta PRISMA (Moher et al. 2009). Para tanto, realizou-se uma varredura bibliográfica em três bases de dados científicas, a citar: a Scientific Electronic Library Online (SciELO); Scopus; Directory of Open Access Journals (DOAJ).

A coleta dos artigos para compor este estudo foi realizada em 21 de julho de 2021, a partir do seguinte conjunto de descritores: "Agricultura 4.0" ou "Agriculture 4.0"; "Tecnologia 4.0” ou “Technology 4.0”; e, "Desenvolvimento Sustentável” ou "Sustainable Development". No recorte temporal foram considerados os últimos dez anos; do ano de 2011 a julho de 2021.

Os critérios de inclusão empregues foram: artigos originais sobre o tema proposto, disponíveis integralmente, em língua portuguesa e inglesa. Foram excluídos todos os trabalhos provenientes de anais de eventos; revisões bibliográficas; fora do recorte temporal e em duplicidade. Inicialmente, os artigos foram filtrados pela leitura independente do título, palavraschave e, por último, pela leitura do resumo. Aqueles que travavam da temática em pauta foram eleitos para leitura dos textos completos.

Foi utilizada para a abordagem qualitativa, a análise de Conteúdo de Bardin (2016). Na qual, os 17 Objetivos do Desenvolvimento Sustentável correspondem às categorias de análise, sendo: C1- erradicação da pobreza; C2- fome zero e agricultura sustentável; C3- saúde e bem-estar; C4- educação de qualidade; C5- igualdade de gênero; C6- água potável e saneamento; C7- energia limpa a acessível; C8- trabalho descente e crescimento econômico; C9- indústria, inovação e infraestrutura; C10-redução das desigualdades; C11- cidades e comunidades sustentáveis; C12- consumo e produção responsáveis; C13- ação contra a mudança climática; C14- vida na água; C15- vida terrestre; C16- paz, justiça e instituições eficazes; C17- parcerias e meios de implementação (ONU, 2015).

Os dados obtidos, foram organizados conforme os seguintes elementos: número anual de artigos publicados, país, autor, nome do periódico, tema central, título e enquadramento dos artigos nas categorias de análise. Além disso, utilizou-se a ferramenta online WordArt.com, para elencar, a frequência de termos presentes no título dos artigos científicos pesquisados.

\section{Resultados e Discussão}

A varredura inicial permitiu identificar 49 estudos, dos quais em uma primeira exclusão, foram removidos 17, por estarem em idioma diferente do estabelecido, duplicados ou correspondiam a resumos de anais de eventos. Nos 31 estudos restantes, realizou-se a leitura completa dos resumos e, em uma segunda exclusão, foram removidos 9 estudos, que se travam de revisão bibliográfica ou abordavam outras temáticas.

Portanto, restaram 23 estudos para serem lidos na íntegra, dos quais 8 foram excluídos, pois, não correspondiam ao objetivo da presente pesquisa. Ao total, apenas 15 estudos foram incluídos na revisão, por atenderem aos critérios de inclusão, conforme se verifica na Figura 1. 
Figura 1. Fluxograma da seleção de estudos para compor a revisão sistemática sobre agricultura digital.

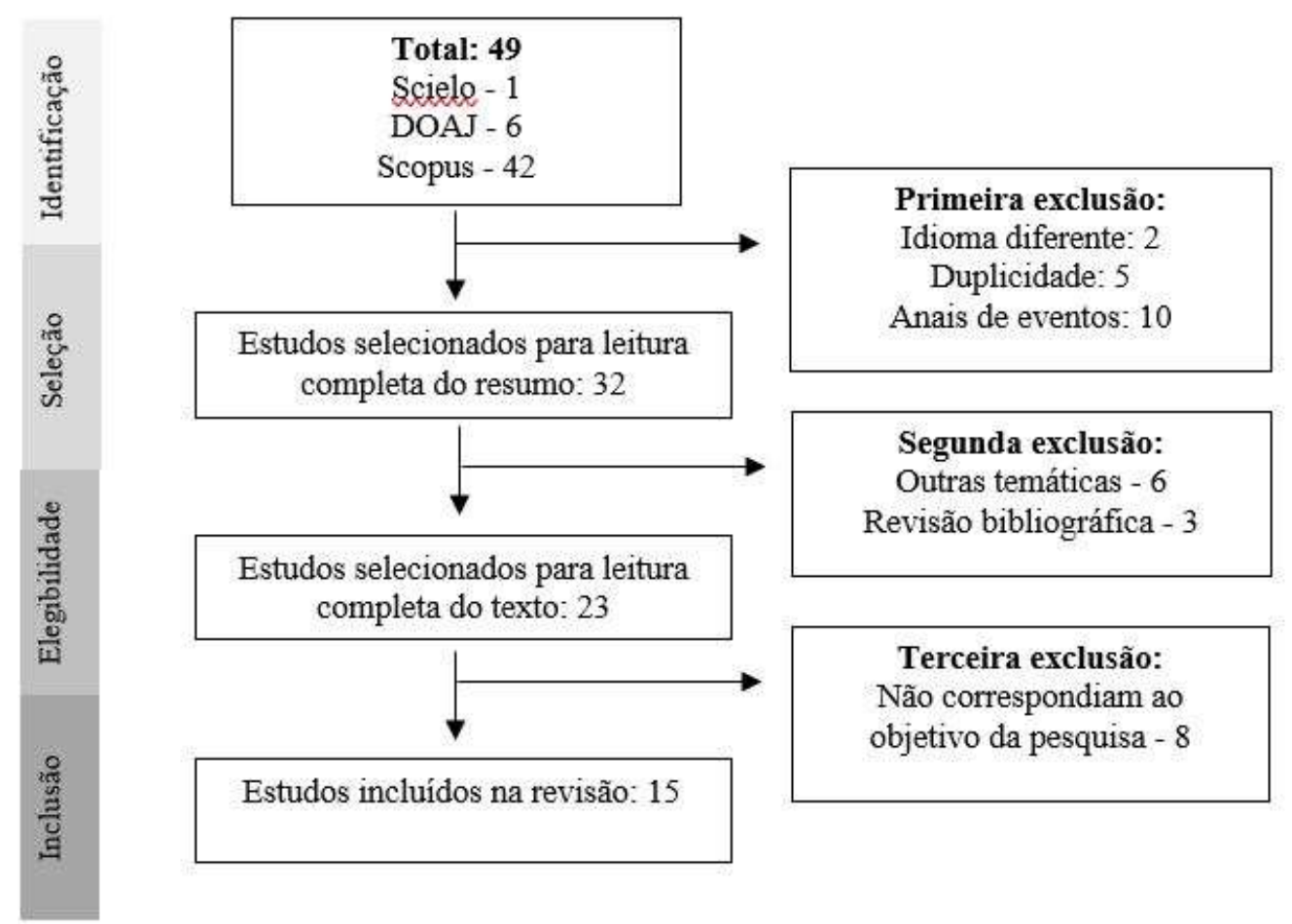

Fonte: Dados da pesquisa (2021).

Os 15 artigos analisados foram publicados em língua inglesa, sendo realizados em diferentes regiões do mundo, a citar: Itália ${ }^{(5)}$, Brasil ${ }^{(3)}$, Espanha ${ }^{(2)}$, Irã ${ }^{(1)}$, Nepal ${ }^{(1)}$, França ${ }^{(1)}$, Malásia ${ }^{(1)}$ e Taiwan ${ }^{(1)}$. Isto indica, portanto, que o uso de tecnologias digitais na agricultura tem sido uma tendência para a superação de desafios em diferentes contextos produtivos.

Os estudos incluídos na presente revisão foram publicados nos anos de $2018^{(1)}, 2019^{(1)}, 2020^{(5)}$ e $2021^{(8)}$. Esses indicativos evidenciam o crescimento de publicações sobre agricultura 4.0 nos últimos anos, em uma lógica de constância em pesquisas desse caráter. Além disso, a disponibilidade dos estudos nas bases de dados consultadas foi desproporcional, sendo a maioria deles encontrados na Scopus (80\%), DOAJ (13,3\%) e SciElo (6,6\%), respectivamente.

Considerando a ampla abordagem entre a agricultura e tecnologia nessas publicações, percebeu-se ênfase aos estudos relacionados com o desenvolvimento de modelos de tecnologia digital para culturas agrícolas específicas, assim como estudos que tencionavam compreender as relações entre o uso e as dificuldades de implementação dessas, no contexto produtivo. Isto posto, na Figura 2, observam-se as palavras mais recorrentes nos títulos dos artigos analisados.

Figura 2. Nuvem de palavras elaborada a partir dos títulos dos artigos pesquisados sobre agricultura digital

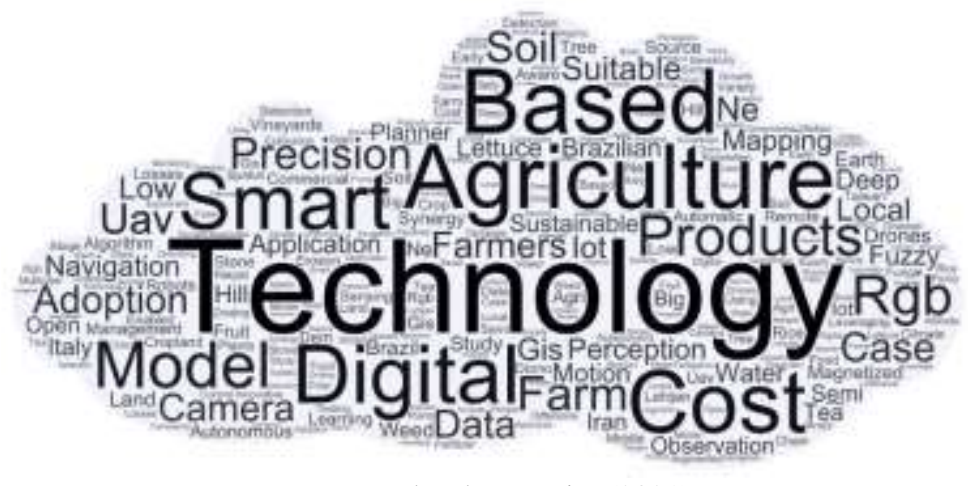

Fonte: Dados da pesquisa (2021). 
Conforme se verifica na Figura 2, além da palavra Tecnologia, recebem destaque as seguintes palavras: agricultura, digital, custo, inteligente, produtos, modelos, sediado (remete ao contexto) e precisão. E, ao amarrar esses conceitos, percebese que os títulos remetem ao uso de tecnologias digitais na agricultura fundamento na conjuntura econômica para o desenvolvimento e a inovação.

Analisando, em vista disso, as abordagens dos estudos, nota-se, ainda, que para além da otimização de custos e melhoria dos processos produtivos, elas se concatenam ao conceito de desenvolvimento sustentável, defendido por Elkington (2012), cujo ressalta a necessidade de envolver as dimensões ambientais, sociais e ambientais.

Apesar disso, esta afirmação, ainda é incompleta, pois, o uso de tecnologias digitais na agricultura, vai muito além desses três aspectos, envolve outras pluralidades políticas, culturais e outras, que se colocam no ambiente agrícola, especialmente dos pequenos produtores rurais. Nesse sentido, é fundamental, repensar o uso das tecnologias digitais na agricultura para o fortalecimento dos sistemas produtivos, de forma que isso inclua, também, os agricultores familiares, oportunizando igualdade de acesso, proporcionando melhoria da qualidade de vida e geração de renda no campo (Massruhá \& Leite 2017).

Acredita-se que agricultura digital poderá beneficiar produtores, consumidores finais e o planeta como um todo. Entretanto, de acordo com Rose et al. (2021) os debates sobre a agricultura 4.0 e suas interfaces ainda estão muito restritas às questões ambientais e de produtividade em sistemas agrícolas muito específicos. Em vista disso, é fundamental uma maior atenção para a sustentabilidade social, implicações éticas, onde os benefícios também possam alcançar, uniformemente produções agrícolas, pessoas e planeta.

Analisando-se os respectivos artigos, constatou-se que eles, em sua maioria externalizaram a necessidade de políticas específicas para expandir o acesso e implementar o uso das tecnologias digitais na agricultura. Justamente, nesta perspectiva, é que se evidencia que a agricultura digital abrange e contempla distintas áreas agrícolas, logo, representa um processo de caráter multidimensional. O Gráfico 1, apresenta as principais áreas abordadas nos estudos pesquisados:

Gráfico 1. Principais áreas envolvidas com as publicações sobre agricultura digital, segundo os estudos pesquisados.

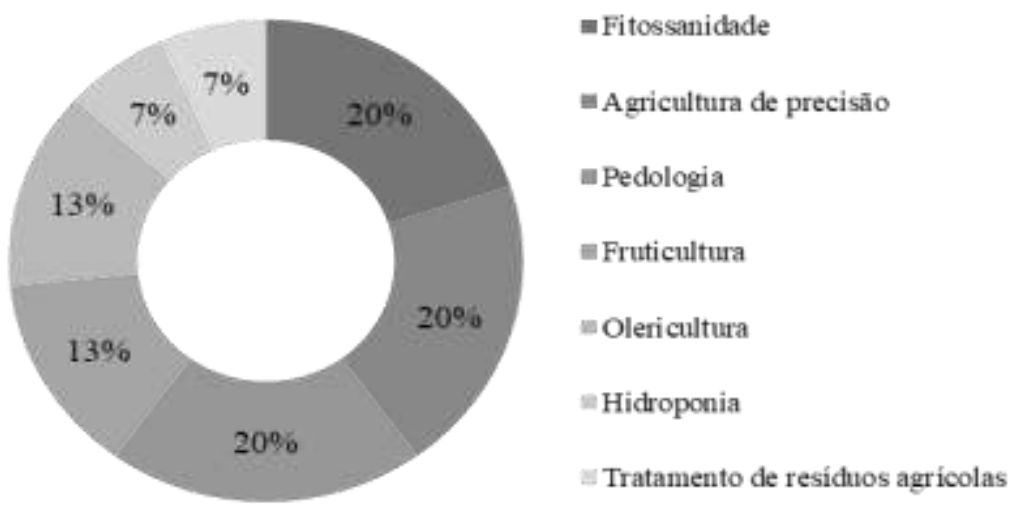

Fonte: Dados da pesquisa (2021).

Verifica-se que há uma diversidade de áreas envolvidas com as produções científicas analisadas, estabelecendo uma interface entre tecnologias digitais e campos de aplicação. As áreas com mais produção são: Fitossanidade; Agricultura de precisão; Pedologia, que juntas representam 60\% da produção. Em seguida, por ordem decrescente têm-se: Fruticultura (13\%), Olericultura (13\%), Hidroponia (7\%) e Tratamento de resíduos agrícolas (7\%). 
O destaque identificado para Fitossanidade, Agricultura de precisão e Pedologia se dá em função de a agricultura digital estar se esforçando no campo da produtividade, visto que esses três fatores são importantes ao crescimento das culturas agrícolas e ao resultado do cultivo, nas diversas regiões do mundo. Ou seja, as informações sobre as características do solo, a sanidade das plantas e o gerenciamento agrícola dos cultivos podem influir na redução de custos, de utilização de produtos químicos e impactos ambientais, fortalecendo a sustentabilidade agrícola (Flores \& Alba, 2014; Salgado \& Cuva, 2019).

Nesse contexto, o desenvolvimento sustentável tem a pretensão de construir uma nova sociedade, com cadeias produtivas que possibilitem a redução das desigualdades, o fortalecimento da justiça social e da equidade, levando em conta a saúde planetária. Ou seja, torna-se "[...] possível desenvolver novos conhecimentos e tecnologias que permitirão o uso mais eficiente dos recursos, ao mesmo tempo que reduzem o impacto ambiental da produção agrícola" (Saccaro Júnior \& Vieira Filho, 2018, p. 5).

É justamente neste âmbito, que se torna possível estabelecer relações sobre agricultura digital e desenvolvimento sustentável. Assim, no Quadro 1 apresentam-se informações dos 15 estudos analisados e seu enquadramento principal nas categorias de análise, que são coerentes aos 17 Objetivos do Desenvolvimento Sustentável da ONU.

Quadro 1. Artigos científicos coletados nas bases de dados SciElo, DOAJ e Scopus sobre o tema agricultura digital, analisados neste estudo e relação com os Objetivos do Desenvolvimento Sustentável (ODS).

\begin{tabular}{|c|c|c|c|c|}
\hline Autor e ano & Periódico & Tema central & Título & ODS \\
\hline $\begin{array}{l}\text { Matulovic et al. } \\
\quad(2021)\end{array}$ & $\begin{array}{l}\text { Acta } \\
\text { Scientiarum. } \\
\text { Agronomy }\end{array}$ & $\begin{array}{l}\text { Modelo que determina a melhor época de } \\
\text { colheita da alface em função do tipo de } \\
\text { água e lâmina de irrigação, melhorando a } \\
\text { produção }\end{array}$ & $\begin{array}{c}\text { Technology } 4.0 \text { with } 0.0 \\
\text { costs: fuzzy model of lettuce } \\
\text { productivity with magnetized water }\end{array}$ & 9 e 12 \\
\hline $\begin{array}{l}\text { Mattivi et al. } \\
\qquad(2021)\end{array}$ & $\begin{array}{l}\text { Remote } \\
\text { sensing }\end{array}$ & $\begin{array}{l}\text { Aeronave não tripulada de baixo custo } \\
\text { que permite o manejo de ervas daninhas } \\
\text { em fazendas de pequeno e médio porte }\end{array}$ & $\begin{array}{l}\text { Can commercial low-cost drones } \\
\text { and open-source gis technologies be } \\
\text { suitable for semi-automatic weed } \\
\text { mapping for smart farming? A case } \\
\text { study in ne italy }\end{array}$ & $\begin{array}{l}3,6,9,11 \\
\text { e } 12\end{array}$ \\
\hline $\begin{array}{l}\text { Safanelli et al. } \\
\qquad(2021)\end{array}$ & Geoderma & $\begin{array}{c}\text { Softwares para o mapeamento e } \\
\text { monitoramento de alta resolução dos } \\
\text { principais atributos da camada superficial } \\
\text { de solos agrícolas }\end{array}$ & $\begin{array}{l}\text { Leveraging the application of Earth } \\
\text { observation data for mapping } \\
\text { cropland soils in Brazil }\end{array}$ & 9 e 12 \\
\hline $\begin{array}{l}\text { Rahimi-Ajdadi \& } \\
\text { Khani (2021) }\end{array}$ & $\begin{array}{c}\text { Remote } \\
\text { Sensing } \\
\text { Applications }\end{array}$ & $\begin{array}{l}\text { Utilização de redes neurais artificiais para } \\
\text { detectar mudanças em áreas agrícolas }\end{array}$ & $\begin{array}{l}\text { Remote sensing-based detection of } \\
\text { tea land losses: the case of lahijan, } \\
\text { iran }\end{array}$ & 9,15 e 17 \\
\hline $\begin{array}{l}\text { Rovira-Más, Saiz- } \\
\text { Rubio \& Cuenca- } \\
\text { Cuenca }(2021)\end{array}$ & $\begin{array}{l}\text { Sensors } \\
\text { Journal }\end{array}$ & $\begin{array}{c}\text { Estratégia de navegação 3D e ultrassom } \\
\text { para guiar um robô ao longo de linhas de } \\
\text { pomar para monitoramento em campo } \\
\text { agrícola }\end{array}$ & $\begin{array}{l}\text { Augmented perception for } \\
\text { agricultural robots navigation }\end{array}$ & 9 e 11 \\
\hline $\begin{array}{l}\text { López-morales et } \\
\text { al. (2021) }\end{array}$ & $\begin{array}{l}\text { Sensors } \\
\text { Journal }\end{array}$ & $\begin{array}{c}\text { Plataforma de Internet das Coisas (IoT) } \\
\text { para avaliar áreas de plantio para } \\
\text { determinadas árvores frutíferas com } \\
\text { caroço }\end{array}$ & $\begin{array}{l}\text { Climate-aware and iot-enabled } \\
\text { selection of the most suitable stone } \\
\text { fruit tree variety }\end{array}$ & 9,11 e 15 \\
\hline Chidi et al. (2021) & $\begin{array}{l}\text { International } \\
\text { Journal of } \\
\text { Geo- } \\
\text { Information }\end{array}$ & $\begin{array}{l}\text { Veículo aéreo não tripulado de alta } \\
\text { resolução (nível centimétrico) para } \\
\text { apresentar características topográficas } \\
\text { precisas de erosão do solo }\end{array}$ & $\begin{array}{l}\text { Sensitivity assessment of spatial } \\
\text { resolution difference in dem for soil } \\
\text { erosion estimation based on uav } \\
\text { observations: an experiment on } \\
\text { agriculture terraces in the middle } \\
\text { hill of nepal }\end{array}$ & 9 e 15 \\
\hline Traversari et al. & Sustainability & $\begin{array}{l}\text { Detecção digital com tecnologias } \\
\text { espectroscópicas e de imagem para }\end{array}$ & $\begin{array}{c}\text { Precision agriculture digital } \\
\text { technologies for sustainable fungal }\end{array}$ & 3,9 e 12 \\
\hline
\end{tabular}




\begin{tabular}{|c|c|c|c|c|}
\hline (2021) & & $\begin{array}{l}\text { identificação de doenças fúngicas } \\
\text { importantes }\end{array}$ & $\begin{array}{l}\text { disease management of ornamental } \\
\text { plants }\end{array}$ & \\
\hline $\begin{array}{l}\text { Chuang, Wang \& } \\
\text { Liou (2020) }\end{array}$ & $\begin{array}{l}\text { International } \\
\text { Journal of } \\
\text { Environmenta } \\
1 \text { Research } \\
\text { and Public } \\
\text { Health }\end{array}$ & $\begin{array}{c}\text { Pesquisa de campo sobre o } \\
\text { conhecimento e as atitudes dos } \\
\text { agricultores em relação às tecnologias } \\
\text { digitais em Taiwan }\end{array}$ & $\begin{array}{l}\text { Farmers' Knowledge, Attitude, and } \\
\text { Adoption of Smart Agriculture } \\
\text { Technology in Taiwan }\end{array}$ & $\begin{array}{c}8,9,10 \mathrm{e} \\
12\end{array}$ \\
\hline $\begin{array}{c}\text { Giannoccaro et al. } \\
(2020)\end{array}$ & $\begin{array}{l}\text { International } \\
\text { Journal of } \\
\text { Precision } \\
\text { Engineering } \\
\text { and } \\
\text { Manufacturin } \\
\text { g }\end{array}$ & $\begin{array}{l}\text { Desenvolvimento de um sistema digital } \\
\text { capaz de controlar com extrema precisão } \\
\text { a quantidade de fertilizante que a cultura } \\
\text { necessita por meio do sistema de } \\
\text { fertirrigação inteligente }\end{array}$ & $\begin{array}{l}\text { A system for optimizing fertilizer } \\
\text { dosing in innovative smart } \\
\text { fertigation pipelines: modeling, } \\
\text { construction, testing and control }\end{array}$ & 3 e 9 \\
\hline Angeloni (2020) & $\begin{array}{l}\text { International } \\
\text { Journal of } \\
\text { Grid and } \\
\text { Utility } \\
\text { Computing }\end{array}$ & $\begin{array}{l}\text { O artigo explica e discute um aparelho } \\
\text { agrícola inovador automático, baseado na } \\
\text { agricultura vertical e na hidroponia }\end{array}$ & Domo farm 4.0 & 2,9 e 12 \\
\hline $\begin{array}{l}\text { Bolfe et al., } \\
\quad(2020)\end{array}$ & Agriculture & $\begin{array}{l}\text { Pesquisa de campo, sobre acesso, uso e } \\
\text { dificuldades na aquisição e } \\
\text { implementação de tecnologias digitais no } \\
\text { Brasil }\end{array}$ & $\begin{array}{l}\text { Precision and Digital Agriculture: } \\
\text { Adoption of Technologies and } \\
\text { Perception of Brazilian Farmers }\end{array}$ & $\begin{array}{c}8,9,10 \mathrm{e} \\
12\end{array}$ \\
\hline $\begin{array}{l}\text { Aghi, Mazzia \& } \\
\text { Chiaberge (2020) }\end{array}$ & Machines & $\begin{array}{l}\text { Desenvolvimento de uma máquina } \\
\text { agrícola de movimento local com baixo } \\
\text { consumo de energia para navegação } \\
\text { autônoma em vinhedos }\end{array}$ & $\begin{array}{l}\text { Local Motion Planner for } \\
\text { Autonomous Navigation in } \\
\text { Vineyards with a RGB-D Camera- } \\
\text { Based Algorithm and Deep } \\
\text { Learning Synergy }\end{array}$ & 3,7 e 9 \\
\hline $\begin{array}{l}\text { Belaud et al. } \\
\quad(2019)\end{array}$ & $\begin{array}{l}\text { Computers in } \\
\text { Industry }\end{array}$ & $\begin{array}{l}\text { Métodos de pré-tratamento da biomassa } \\
\text { lignocelulósica na cadeia de } \\
\text { abastecimento do arroz, para melhoria da } \\
\text { gestão da sustentabilidade }\end{array}$ & $\begin{array}{c}\text { Big data for agri-food } 4.0 \text { : } \\
\text { Application to sustainability } \\
\text { management for by-products supply } \\
\text { chain }\end{array}$ & $\begin{array}{c}6,9,12 \mathrm{e} \\
15\end{array}$ \\
\hline $\begin{array}{l}\text { Norasma et al. } \\
\text { (2018) }\end{array}$ & $\begin{array}{l}\text { Earth } \\
\text { Environmenta } \\
1 \text { Science }\end{array}$ & $\begin{array}{c}\text { Veículo aéreo não tripulado capaz de } \\
\text { monitorar o estágio mais adequado para a } \\
\text { colheita de arroz }\end{array}$ & $\begin{array}{l}\text { Rice crop monitoring using } \\
\text { multirotor uav and rgb digital } \\
\text { camera at early stage of growth }\end{array}$ & 9,11 e 12 \\
\hline
\end{tabular}

Fonte: Dados da pesquisa (2021).

O Quadro 1 demonstra que as tecnologias digitais vêm sendo empregadas para diversas finalidades, na maioria dos artigos pesquisados, fortalecendo seu caráter abrangente, dentro dos contextos produtivos. Também foi possível constatar que todos os artigos se relacionam com um ou mais dos Objetivos do Desenvolvimento Sustentável. No entanto, de forma unanime todos os estudos se relacionam com a meta 9, que têm como principal objetivo a construção de processos produtivos inclusivos, sustentáveis, resilientes, com enfoque na inovação (ONU, 2015). Neste caso, a inovação tem grande relação com o digital na agricultura, sendo esse um dos principais diferenciais hoje.

Para além, a transformação digital nas práticas agrícolas, pode, de forma indireta, colaborar com vários outros Objetivos do Desenvolvimento Sustentável e "[...] pode contribuir significativamente para o alcance desses objetivos, em especial na redução da fome, saúde e bem-estar, emprego digno e crescimento econômico, redução das desigualdades, consumo e produção responsáveis, combate às alterações climáticas, vida sobre a terra, paz, justiça e instituições fortes" (Bolfe et al., 2020, p. 382).

Outro dado que muito chamou atenção, foi a quantidade de artigos publicados em 2021, onde se constatou uma maior diversificação e pluralidade de estudos, evidenciando que mais da metade (53\%) dos estudos foram publicados recentemente. 
Isso demonstra o acirrado debate e corrida tecnológica na agricultura, que tem fortalecido o interesse de pesquisadores no estudo e desenvolvimento de modelos digitais para distintas culturas agrícolas.

Além disso, a agricultura digital pode abrir novos caminhos para médios e pequenos produtores rurais, uma vez que ela pode aproximá-los de mercados, reduzindo custos processuais e na superação de disparidades de escala (Buainain; Cavalcante \& Consoline, 2021). Contudo, a questão atual consiste em repensar questões de acesso a elas, disponibilidade de infraestrutura e assistência técnica, para as distintas realidades do planeta.

\section{Considerações Finais}

Neste estudo, buscamos demonstrar de que forma o tema, agricultura digital vem sendo descrito em artigos científicos publicados ao redor do mundo e sua relação com os Objetivos do Desenvolvimento Sustentável, da ONU. Verificou-se que, em todos os artigos, há relação com as metas da ONU, em especial com a meta 9 , estando eles diretamente associadas às inovações tecnológicas para a produção de alimentos e aprimoramento de técnicas de manejo.

Além disso, os resultados obtidos não estavam relacionados apenas a fatores de produtividade, custos ou estratégias de precisão. Os estudos analisados, portanto, indicam que os benefícios da agricultura digital se externalizam com a qualidade de vida do produtor e pode atender à sustentabilidade dentro dos processos agrícolas.

Em vista disso, os estudos científicos sobre agricultura digital e Objetivos do Desenvolvimento Sustentável demonstram seu caráter interdisciplinar e transversal, alinhados as demandas atuais de estabelecimento de novas relações entre sociedade e ambiente. Nossos resultados, apontam ainda para evidências da necessidade de formulação de políticas públicas eficientes, de incentivo e continuidade, para grandes e pequenos produtores; sugerem que esta é um dos principais aspectos a serem considerados, tendo em vista o estabelecimento agrícola, sua cultura e modo de produção. A ideia não é generalizar, pelo contrário, é ofertar as tecnologias digitais dentro da realidade de cada unidade produtiva.

Contudo, ainda, constataram-se algumas limitações. Embora nossa busca fosse apoiada em bases de dados mundiais, a coleta ainda não permitiu captar todos os estudos disponíveis. Por conseguinte, sugerem-se que estudos futuros, incluam um trabalho de coleta de dados em uma maior quantidade de base de dados, projetado para avaliar de forma ampla a relação da agricultura 4.0 com os Objetivos do Desenvolvimento Sustentável e com a melhoria da qualidade ambiental.

\section{Agradecimentos}

Agradecemos a Universidade Estadual do Oeste do Paraná (UNIOESTE), a Coordenação de Aperfeiçoamento de Pessoal de Nível Superior (CAPES) e o Conselho Nacional de Desenvolvimento Científico e Tecnológico (CNPq), pelo financiamento por meio da bolsa concedida.

\section{Referências}

Aghi, D., Mazzia, V. \& Chiaberge, M. (2020). Local Motion Planner for Autonomous Navigation in Vineyards with a RGB-D Camera-Based Algorithm and Deep Learning Synergy. Machines, 8 (2), 1-16.

Angeloni, S. (2020). Domo Farm 4.0. International Journal of Grid and Utility Computing, 11 (2), 135-142.

Bardin, L. (2016). Análise de conteúdo. Edições 70.

Belauda, J. P., Prioux, N., Vialle, C. \& Sablayrolles, C. (2019). Big data for agri-food 4.0: Application to sustainability management for by-products supply chain. Computers and Industry, 111 (1), 41-50.

Bolfe, E. L., Jorge, L. A. C., Sanches, I. D., Luchiari Júnior, A., Costa, C. C., Victoria, D. C., Inamasu, R. Y., Grego, C. R., Ferreira, V. R. \& Ramirez, A. R. (2020). Precision and Digital Agriculture: Adoption of Technologies and Perception of Brazilian Farmers. Agriculture, 10 (12), 1-16. 
Bolfe, E. L., Barbedo, J. G. A., Massruhá, S. M. F. S., Souza, K. X. S. \& Assad, E. D. (2020). Desafios, tendências e oportunidades em agricultura digital no Brasil. In: Massruhá, S. M. F. S., Leite, M. A. A., Oliveira, S. R. M., Meira, C. A. A., Luchiari Junior, A. \& Bolfe, E. L. (2020). Agricultura digital: pesquisa, desenvolvimento e inovação nas cadeias produtivas. Brasília: Embrapa, 380-406.

Buainain, A. M., Cavalcante, P. \& Consoline, L. (2021). Estado atual da agricultura digital no Brasil: Inclusão dos agricultores familiares e pequenos produtores rurais. Santiago: CEPAL.

Chidi, C. L., Zhao, W., Chaudhary, S., Xiong, D. \& Wu, Y. (2021). Sensitivity Assessment of Spatial Resolution Difference in DEM for Soil Erosion Estimation Based on UAV Observations: An Experiment on Agriculture Terraces in the Middle Hill of Nepal. International Journal of Geo-Information, 10 $(1), 1-17$.

Chuang, J. H., Wang, J. H. \& Liou, Y. C. (2020). Farmers' Knowledge, Attitude, and Adoption of Smart Agriculture Technology in Taiwan. International Journal of Environmental Research and Public Health, 17 (7236), 1-8.

Clercq, M., Vats, A. \& Biel, A. (2018). Agriculture 4.0: The future of farming technology. World Government Summit.

Elkington, J. (2012). Sustentabilidade: canibais com garfo e faca. M Books do Brasil.

Flores, C. A. \& Alba, J. M. F. (2014). A Pedologia e a Agricultura de Precisão. In: Bernardi, A. C. C., Naime, J. M., Resende, A. V., Bassoi, L. H. \& Inamasu, R. Y. (Orgs.) Agricultura de precisão: resultados de um novo olhar. Embrapa, 36-47.

Giannoccaro, N. I., Persico, G., Strazzella, S., Lay-Ekuakille, A. \& Visconti, P. (2020). A System for Optimizing Fertilizer Dosing in Innovative Smart Fertigation Pipelines: Modeling, Construction, Testing and Control. International Journal of Precision Engineering and Manufacturing, 21 (1), $1581-1596$.

López-Morales, J. Á., Martínez, J. Á., Caro, M., Erena, M. \& Skarmeta, A. F. (2021). Climate-Aware and IoT-Enabled Selection of the Most Suitable Stone Fruit Tree Variety. Sensors Journal, 21 (3867) 1-27.

Massruhá, S. M. F. S. \& Leite, M. A. A. (2017). Agro 4.0 - rumo à agricultura digital. In: Magnoni Júnior, L., Stevens, D., Silva, W. T. L., Vale, J. M. F., Purini, S. R. M., Magnoni, M. G. M., Sebastião, E., Branco Júnior, G., Adorno Filho, E. F., Figueiredo, W. S. \& Sebastião, I. (Orgs.). JC na Escola Ciência, Tecnologia e Sociedade: mobilizar o conhecimento para alimentar o Brasil. (2a ed.), Centro Paula Souza. 28-35

Mattivi, P., Pappalardo, S. E., Nikolic, N., Mandolesi, L., Persichetti, A., Marchi, M. \& Masin, R. (2021). Can Commercial Low-Cost Drones and OpenSource GIS Technologies Be Suitable for Semi-Automatic Weed Mapping for Smart Farming? A Case Study in NE Italy. Remote sensing, 13 (1869), 1-21.

Matulovic, M., Putti, F. F., Cremasco, C. P. \& Gabriel filho, L. R. A. (2021). Technology 4.0 with 0.0 costs: fuzzy model of lettuce productivity with magnetized water. Acta Scientiarum Agronomy, 43 (1), 1-15.

Moher, D., Liberati, A., Tetzlaff, J. \& Altman, D. G. (2009). Preferred reporting items for systematic reviews and meta-analyses: the PRISMA statement. PloS Med, 6 (7), 2-9.

Norasma, C. Y. N., Abu Sari, M. Y., Fadzilah, M. A., Ismail, M. R., Omar, M. H., Zulkamari, B., Hassim, Y. M. M. \& Tarmidi, Z. (2018). Rice crop monitoring using multirotor UAV and RGB digital camera at early stage of growth. Earth Environmental Science, 169 (1), $24-25$.

ONU. Organização das Nações Unidas. (2015). Agenda 2030. UNDP.

Rahimi-Ajdadi, F. \& Khani, M. (2021). Remote sensing-based detection of tea land losses: The case of Lahijan, Iran. Remote Sensing Applications: Society and Environment, 23 (568) 1-9.

Rose, D. C., Wheeler, R., Winter, M., Lobley, M. \& Chivers, C. A. (2021). Agriculture 4.0: Making it work for people, production, and the planet. Land Use Policy, 100 (1), 1-5.

Rovira-Mas, F., Saiz-Rubio, V. \& Cuenca-Cuenca, A. (2021). Augmented Perception for Agricultural Robots Navigation. Sensors Journal, 21 (10), 1171211727.

Saccaro Júnior, N. L. \& Vieira Filho, J. E. R. (2018). Agricultura e sustentabilidade: esforços brasileiros para mitigação dos problemas climáticos. IPEA.

Salgado, T. P. \& Kuva, M. A. (2019). Levantamento de problemas fitossanitários e tomada de decisão. Universidade Estadual Paulista.

Traversari, S., Cacini, S., Galieni, A., Nesi, B., Nicastro, N. \& Pane, C. (2021). Precision Agriculture Digital Technologies for Sustainable Fungal Disease Management of Ornamental Plants. Sustainability, 13 (3707), 1-22.

Safanelli, J. L., Demattê, J. A. M., Chabrillat, S., Poppiel, R. R., Rizzo, R., Dotto, A. C., Silvero, N. E. Q., Mendes, W. S., Bonfatti, B. R., Ruiz, L. F. C., Caten, A. \& Dalmolin, R. S. D. (2021). Leveraging the application of Earth observation data for mapping cropland soils in Brazil. Geoderma, 396 (15), 1-13. 